\title{
IDEALS GENERATED BY QUADRATIC POLYNOMIALS
}

\author{
Tigran Ananyan And Melvin Hochster
}

\begin{abstract}
Let $R$ be a polynomial ring in $N$ variables over an arbitrary field $K$ and let $I$ be an ideal of $R$ generated by $n$ polynomials of degree at most 2 . We show that there is a bound on the projective dimension of $R / I$ that depends only on $n$, and not on $N$. The proof depends on showing that if $K$ is infinite and $n$ is a positive integer, there exists a positive integer $C(n)$, independent of $N$, such that any $n$ forms of degree at most 2 in $R$ are contained in a subring of $R$ generated over $K$ by at most $t \leq C(n)$ forms $G_{1}, \ldots, G_{t}$ of degree 1 or 2 such that $G_{1}, \ldots, G_{t}$ is a regular sequence in $R$. $C(n)$ is asymptotic to $2 n^{2 n}$.
\end{abstract}

\section{Introduction}

Throughout this paper let $R$ denote a polynomial ring over an arbitrary field $K$ : say $R=K\left[x_{1}, \ldots, x_{N}\right]$. We will denote the projective dimension of the $R$-module $M$ over $R$ by $\operatorname{pd}(M)$. The following conjecture was posed by Peeva and Stillman [10].

Conjecture 1.1. There is an upper bound, independent of $N$, on $\operatorname{pd}(R / I)$, where $I$ is any ideal of $R$ generated by $n$ homogeneous polynomials of given degrees $d_{1}, \ldots, d_{n}$.

One motivation for proving this conjecture comes from its equivalence to the following open question (the proof of equivalence due to Caviglia is given in [4]):

Conjecture 1.2. There is a bound on the Castelnuovo-Mumford regularity of an ideal in polynomial ring that depends only on the number of its minimal generators and the degrees of those generators.

Another motivation for Conjecture 1.1 comes from certain results about ideals generated by three homogeneous polynomials. A construction of Burch [2] in the local case, extended to the global case by Kohn [7], shows that there exist three generated ideals of arbitrarily large projective dimension. However in the polynomial ring case, as the projective dimension grows, the degrees of the generators are growing as well, thus motivating Conjecture 1.1 (see [4] for analysis of growth of degrees in Burch's construction).

Conjecture 1.1 clearly holds when $n \leq 2$ or when all of the $d_{i}=1$, but even the simplest next case of $n=3$ and $d_{1}=\bar{d}_{2}=d_{3}=2$ requires non-trivial arguments. D. Eisenbud and C. Huneke proved that if $I$ is generated by three quadratic forms, then $\operatorname{pd}(R / J) \leq 4$. Engheta $[5,6]$ showed the existence of a bound on the projective dimension for three cubic forms; he has shown that $\operatorname{pd}(R / I) \leq 36$ (even though in all known examples of three cubics $\operatorname{pd}(R / I) \leq 5)$. In general, the projective dimension

Received by the editors 12 August 2011.

Key words and phrases. polynomial ring, ideal, quadratic polynomials, projective dimension, regular sequence. 
can grow relatively fast as one increases the number of generators and the degrees (see $[1,3,9]$ for specific examples). In this paper the authors prove Conjecture 1.1 for the case when all of the $d_{i}$ are at most 2 and $n$ is arbitrary.

Since a base change of the field $K$ to a larger field does not affect the projective dimension, in the remainder of this paper we often pass to the case where $K$ is infinite.

\section{The main results}

We use recursion on $h$ to define a function $B(m, n, h)$ of three non-negative integers, with $h \leq n$, as follows. Let $B(m, n, 0)=m+m n=m(n+1)$. If $h \geq 1$, let

$$
B(m, n, h)=(m+h)\left(n^{3}+n^{2}+n+1\right)+h(n+1)+B\left((m+h) n^{2}, n, h-1\right) .
$$

We also define $C(s)$ to be the largest value of $B(m, n, h)+h$ for $m+n=s$ and $0 \leq h \leq n-1$. Let $C_{0}(s)$ be the largest value of $B(0, n, h)+m+h$ for $m+n=s$ and $0 \leq h \leq n-1$. It is shown in Section 6 that both $C_{0}(s)$ and $C(s)$ are asymptotic to $2 s^{2 s}$.

Our goal is to prove the following result.

Main Theorem 2.1. Let $R=K\left[x_{1}, \ldots, x_{N}\right]$ be a polynomial ring over a field $K$. Let $F_{1}, \ldots, F_{m+n}$ be forms of degree at most 2 generating ideal $I$, and suppose that the linear forms among them span a $K$-vector space of dimension $m$. Let $h$ denote the height of the ideal generated by the images of the quadratic $F_{j}$ in the ring obtained from $R$ by killing the ideal generated by all of the $F_{i}$ that are linear.

If $K$ is infinite, after a linear change of variables, there are at most $b \leq B(m, n, h)$ variables $y_{1}, \ldots, y_{b}$ and at most $c \leq h$ quadratic forms $G_{1}, \ldots, G_{c}$ in $I$ such that

(1) The elements $y_{1}, \ldots, y_{b}, G_{1}, \ldots, G_{c}$ form a regular sequence in $R$.

(2) The $F_{i}$ are in the polynomial ring $K\left[y_{1}, \ldots, y_{b}, G_{1}, \ldots, G_{c}\right]$.

(3) The quadratic forms $G_{1}, \ldots, G_{c}$ are also a regular sequence modulo those generators of $I$ that involve only the variables $y_{1}, \ldots, y_{b}$.

(Condition (3) is automatic: see Lemma 3.2 below.)

Hence, if $K$ is infinite, the elements $F_{1}, \ldots, F_{m+n}$ are contained in the $K$-subalgebra of $R$ generated by a regular sequence of at most $B(m, n, h)+h$ linear and quadratic forms. It also follows that, if $K$ is infinite, the elements $F_{1}, \ldots, F_{m+n}$ are contained in the $K$-subalgebra of $R$ generated by a regular sequence of at most $C(m+n)$ forms of degree at most 2.

Therefore, for every field $K$, the projective dimension of $R / I$ is at most $B(0, n, h)+$ $m+h$, and is also at most $C_{0}(m+n)$.

Note that the last statement follows from the first because the projective dimension drops by $m$ when we kill $m$ variables in $I$ and pass to the polynomial ring in the remaining variables, replacing the original $F_{j}$ by the images of the quadratic $F_{j}$ in the smaller polynomial ring.

Note also that if $h=n$ then there is a much better result, in that $F_{1}, \ldots, F_{m+n}$ is already a regular sequence.

Observe also that, as mentioned earlier, the projective dimension does not change when we enlarge the base field. 
Given polynomials of degree at most 2 that need not be forms, each is the sum of at most one quadratic form, one linear form, and a scalar that is already in $K$. Hence:

Main Theorem 2.2. Let $R=K\left[x_{1}, \ldots, x_{N}\right]$ be a polynomial ring over a field $K$. Let $F_{1}, \ldots, F_{s}$ be polynomials of degree at most 2 , and let $I=\left(F_{1}, \ldots, F_{s}\right)$. If $K$ is infinite, the elements $F_{1}, \ldots, F_{s}$ are contained in the $K$-subalgebra of $R$ generated by a regular sequence of at most $C(2 s)$ forms of degree at most 2 . Consequently, for any field $K$, the projective dimension of $R / I$ is at most $C(2 s)$.

Finally, observe that these theorems hold even if we allow the ambient polynomial ring to have a set of variables of arbitrary infinite cardinality, because any given finite set of polynomials will only involve finitely many of the variables.

\section{Preliminary results}

To prove these theorems, we will need the following four lemmas.

Lemma 3.1. Let $I$ be a homogeneous ideal in the polynomial ring $R$. If we take the image of $I$ after killing some of the variables, its height does not increase. Much more generally, let $R$ be a locally equidimensional catenary Noetherian ring, let $x$ be any element not in any minimal prime of $R$, and let $I$ be any ideal such that for every minimal prime $P$ of $I, P+x R \neq R$. Then the height of $I(R / x R)$ is at most the height of $I$.

Proof. The first statement follows from the second by induction on the number of variables killed. Note that when $I$ is proper homogeneous, so are all of its minimal primes, and this is preserved both by adding $x R$ for a variable $x$ and by killing $x R$. Without loss of generality, we may replace the ideal $I$ by a minimal prime $P$ of the same height. Since $R$ is locally equidimensional and catenary, the proper ideal $P+x R$ has height equal to either ht $(P)$ or ht $(P)+1$, depending on whether $x \in P$ or not: this can be checked after localizing at any minimal prime of $P+x R$. After $x R$ is killed, the height drops by 1 , because $x$ is not in any minimal prime of $R$.

Lemma 3.2. If $F_{r+1}, \ldots, F_{r+s}$ form a regular sequence modulo a set of variables containing the variables occurring in $F_{1}, \ldots, F_{r}$, then they form a regular sequence modulo the ideal $\left(F_{1}, \ldots, F_{r}\right)$.

Proof. The variables killed generate a $\operatorname{ring} A$, and we may form the quotient ring $B=A /\left(F_{1}, \ldots, F_{r}\right) A$. The polynomial ring $C$ in the rest of the variables over $B$ is flat over $B$. It suffices to prove the statement after replacing $B, C$ by their localizations at their homogeneous maximal ideals. But then the result follows from $[8$, Ch. 8 , (20.F)]: if the image of a sequence in the closed fiber of a flat local extension is regular, so is the original sequence.

A regular sequence of forms in a polynomial ring over a field $K$ can be extended to a homogeneous system of parameters. The original polynomial ring is module finite and free over the polynomial ring generated over $K$ by the homogeneous system of parameters. Hence: 
Lemma 3.3. Let $F_{1}, \ldots, F_{t}$ be a regular sequence of forms in a polynomial ring $R$ over a field $K$. Then $R$ is free, hence, faithfully flat over $A=K\left[F_{1}, \ldots, F_{t}\right]$. Hence, for any ideal $J$ of $R$ whose generators lie in $A, p d(R / J) \leq t$.

Lemma 3.4. Let $K$ be any field, let $y_{1}, \ldots, y_{r}, z_{1}, \ldots, z_{s}$ be $r+s$ indeterminates over $K$, let $\alpha_{1}, \ldots, \alpha_{n} \in K\left[y_{1}, \ldots, y_{r}\right]$, and let $\beta_{1}, \ldots, \beta_{n} \in K\left[z_{1}, \ldots, z_{s}\right]$. Then we can map the polynomial ring $K\left[T_{1}, \ldots, T_{n}\right]$ onto $K\left[\alpha_{1}, \ldots, \alpha_{n}\right]$ as a $K$-algebra so that each $T_{i} \mapsto \alpha_{i}$. Call the kernel $P$. Likewise, we can map the polynomial ring $K\left[T_{1}, \ldots, T_{n}\right]$ onto $K\left[\beta_{1}, \ldots, \beta_{n}\right]$ as a $K$-algebra so that each $T_{i} \mapsto \beta_{i}$. Suppose that the kernel of this map is also $P$. Finally, we can map the polynomial ring $K\left[T_{1}, \ldots, T_{n}\right]$ onto

$$
K\left[\alpha_{1}+\beta_{1}, \ldots, \alpha_{n}+\beta_{n}\right] \subseteq K\left[y_{1}, \ldots, y_{r}, z_{1}, \ldots, z_{s}\right]
$$

as a K-algebra so that each $T_{i} \mapsto \alpha_{i}+\beta_{i}$. Suppose that the kernel of this map contains $P$. Assume also that $P$ is homogeneous, which is automatic if the $\alpha_{i}$ (or the $\beta_{i}$ ) are homogeneous of the same degree over $K$. Then $P$ is generated by linear forms over $K$.

Proof. Let $L$ denote an algebraic closure of $K$. Since

$$
0 \rightarrow P \rightarrow K\left[T_{1}, \ldots, T_{n}\right] \rightarrow K\left[\alpha_{1}, \ldots, \alpha_{n}\right] \rightarrow 0
$$

is exact and $K\left[\alpha_{1}, \ldots, \alpha_{n}\right] \rightarrow K\left[y_{1}, \ldots, y_{r}\right]$ is injective, the same holds after we apply $L \otimes_{K} \ldots$. Similar remarks apply when we consider the $K$-algebra spanned by the $\beta_{i}$. It follows that $L \otimes_{K} P$, which may be identified with expansion of $P$ to $L\left[y_{1}, \ldots, y_{r}\right]$, is the kernel of the map of $L\left[T_{1}, \ldots, T_{n}\right]$ onto $L\left[\alpha_{1}, \ldots, \alpha_{n}\right]$, and taken together with the corresponding facts for the $\beta_{i}$ and the $\alpha_{i}+\beta_{i}$, this shows that our hypotheses are preserved after base change to $L$. If we know the result for $L$, we know that $L \otimes_{K} P$ is generated as an ideal by its degree 1 part, $\left[L \otimes_{K} P\right]_{1}=L \otimes_{K}[P]_{1}$. Then $[P]_{1} K\left[T_{1}, \ldots, T_{n}\right]$ expands to $P L\left[T_{1}, \ldots, T_{n}\right]$. Since $L\left[T_{1}, \ldots, T_{n}\right]$ is faithfully flat over $K\left[T_{1}, \ldots, T_{n}\right]$, the contractions $[P]_{1} K\left[T_{1}, \ldots, T_{n}\right]$ and $P$, respectively, are equal. That is, $P$ is generated by $[P]_{1}$.

Hence, we may assume without loss of generality that $K$ is algebraically closed. Consider the algebraic set $V$ defined by $P$ in affine $n$-space over $K . V$ is closed under scalar multiplication because $P$ is homogeneous. We claim that $V$ is closed under addition. For suppose that $\left(c_{1}, \ldots, c_{n}\right),\left(d_{1}, \ldots, d_{n}\right) \in V$. Then there is a $K$-homomorphism $K\left[\alpha_{1}, \ldots, \alpha_{n}\right] \rightarrow K$ sending each $\alpha_{i} \rightarrow c_{i}$ and, similarly, a $K$-homomorphism $K\left[\beta_{1}, \ldots, \beta_{n}\right] \rightarrow K$ sending every $\beta_{i} \rightarrow d_{i}$. This yields a $K$ homomorphism $\theta: K\left[\alpha_{1}, \ldots, \alpha_{n}\right] \otimes_{K} K\left[\beta_{1}, \ldots, \beta_{n}\right] \rightarrow K$ such that for every $i$, $\alpha_{i} \mapsto c_{i}$ and $\beta_{i} \mapsto d_{i}$. We may tensor the injections $K\left[\alpha_{1}, \ldots, \alpha_{n}\right] \subseteq K\left[y_{1}, \ldots, y_{r}\right]$ and $K\left[\beta_{1}, \ldots, \beta_{n}\right] \subseteq K\left[z_{1}, \ldots, z_{s}\right]$ over $K$ to obtain an injection

$$
K\left[\alpha_{1}, \ldots, \alpha_{n}\right] \otimes_{K} K\left[\beta_{1}, \ldots, \beta_{n}\right] \subseteq K\left[y_{1}, \ldots, y_{r}, z_{1}, \ldots, z_{s}\right]
$$

that enables us to identify $K\left[\alpha_{1}, \ldots, \alpha_{n}\right] \otimes_{K} K\left[\beta_{1}, \ldots, \beta_{n}\right]$ with its image

$$
K\left[\alpha_{1}, \ldots, \alpha_{n}, \beta_{1}, \ldots, \beta_{n}\right] \subseteq K\left[y_{1}, \ldots, y_{r}, z_{1}, \ldots, z_{s}\right] .
$$

Hence, we may think of $\theta$ as a $K$-homomorphism $K\left[\alpha_{1}, \ldots, \alpha_{n}, \beta_{1}, \ldots, \beta_{n}\right] \rightarrow K$ such that for every $i, 1 \leq i \leq n, \alpha_{i}+\beta_{i} \mapsto c_{i}+d_{i}$. Since every polynomial $H \in P$ vanishes on $\left(\alpha_{1}+\beta_{1}, \ldots, \alpha_{n}+\beta_{n}\right)$, we have that $H\left(c_{1}+d_{1}, \ldots, c_{n}+d_{n}\right)=0$ as 
well. This completes the proof that $V$ is closed under addition, and so $V$ is a vector subspace of $K^{n}$. But then its defining prime ideal $P$ is generated by linear forms.

\section{The basic step: putting the $F_{i}$ and variables in standard form}

We are going to make iterative use of the construction of putting the $F_{i}$ and the variables in standard form described just below.

We shall say that the $F_{i}$ and the variables $x_{j}$ are in standard form if the following conditions hold:

(1) $F_{n+i}=x_{i}$ for $1 \leq i \leq m$ and the remaining $F_{i}$ are quadratic or 0 .

(2) No $F_{i}$ for $1 \leq i \leq n$ has a monomial term in $K\left[x_{1}, \ldots, x_{m}\right]$.

(3) $F_{1}, \ldots, F_{h}, x_{1}, \ldots, x_{m}, x_{m+h+1}, \ldots, x_{N}$ is a regular sequence.

(4) There is a non-negative integer $r \leq(m+h) n$ such that when the $F_{i}$ are written as polynomials in the variables $x_{1}, \ldots, x_{h+m}$ with coefficients in the ring $K\left[x_{h+m+1}, \ldots, x_{N}\right]$, the coefficients occurring for the variables $x_{1}, \ldots, x_{h+m}$ are in the $K$-span of $x_{h+m+1}, \ldots, x_{h+m+r}$.

(5) There is a non-negative integer $s \leq(m+h) n^{2}$ such that when the $F_{i}$ are written as polynomials in the variables $x_{1}, \ldots, x_{h+m+r}$ with coefficients in the ring $K\left[x_{h+m+r+1}, \ldots, x_{N}\right]$, the coefficients occurring for the variables $x_{h+m+1}, \ldots$, $x_{h+m+r}$ are in the $K$-span of $x_{h+m+r+1}, \ldots, x_{h+m+r+s}$.

(6) We call $x_{m+1}, \ldots, x_{m+h}$ front variables. We shall also denote them $u_{1}, \ldots, u_{h}$. Let $f_{i}$ denote the image of $F_{i}$ under the $K$-homomorphism $\pi: R \rightarrow K\left[u_{1}, \ldots, u_{h}\right]$ that kills all the variables except the front variables while fixing the front variables (note that $\pi$ kills $F_{i}$ for $i>n$ ). Then there is an integer $d, h \leq d \leq n$, such that $f_{1}, \ldots, f_{d}$ are linearly independent over $K$ while $f_{i}=0$ for $i>d$. We call $f_{1}, \ldots, f_{n}$ the front polynomials.

Under these conditions we call $x_{1}, \ldots, x_{m}$ leading variables, and, as already mentioned, we call $x_{m+1}, \ldots, x_{m+h}$ the front variables, and denote them $u_{1}, \ldots, u_{h}$. We call $x_{h+m+1}, \ldots, x_{h+m+r}$ the primary coefficient variables and use the alternative notation $\underline{v}=v_{1}, \ldots, v_{r}$ for them. We call $x_{h+m+r+1}, \ldots, x_{h+m+r+s}$ the secondary coefficient variables and we write $\underline{w}=w_{1}, \ldots, w_{s}$ as an alternative notation for them. We refer to $x_{h+m+r+1}, \ldots, x_{N}$ as the tail variables. We use the alternative notation $\underline{z}=z_{1}, \ldots, z_{N-(h+m+r)}$ for the tail variables. It is very important to note that the tail variables include the secondary coefficient variables $\underline{w}$.

Let $\tau$ denote the $K$-homomorphism from $R$ to the polynomial ring in the tail variables that fixes the tail variables and kills the others. We write $g_{i}=\tau\left(F_{i}\right)$. Again, $\tau$ kills $F_{i}$ for $i>n$. We call $g_{1}, \ldots, g_{n}$ the tail polynomials.

We can map the polynomial ring $K\left[T_{1}, \ldots, T_{n}\right]$ onto $K\left[f_{1}, \ldots, f_{n}\right]$ by $T_{i} \mapsto f_{i}$. The kernel is a prime ideal $P$ in $K\left[T_{1}, \ldots, T_{n}\right]$, which we call the ideal of front relations.

We want to see that these conditions can be achieved. Note, however, that standard form is far from unique.

4.1. Achieving standard form. We want to show that the sequence can be put in standard form without changing the ring it generates. 
If the $F_{i}$ are linearly dependent over $K$, one or more $F_{i}$ may be replaced by 0 , and we assume that any 0 elements occur in the final spots among the first $n$ terms of the sequence. Hence, we may assume that the linear forms that occur are linearly independent. We may assume that the $F_{i}$ are numbered so that any linear forms occur as a final segment, and we may assume these forms are also an initial segment of the variables. Thus, we have $x_{i}=F_{n+i}, 1 \leq i \leq m$.

We may now subtract from each $F_{i}, 1 \leq i \leq n$, the sum of all terms that occur and involve only $x_{1}, \ldots, x_{m}$.

Since $K$ is infinite, after replacing $F_{1}, \ldots, F_{h}$ by suitably general linear combinations of the quadratic forms $F_{1}, \ldots, F_{n}$, we may assume that the images of $F_{1}, \ldots, F_{h}$ form a maximal regular sequence in $\left(F_{1}, \ldots, F_{n}\right) R /\left(x_{1}, \ldots, x_{m}\right) R$. Note that for $i \leq n$, every $F_{i}$ will be quadratic or else 0 . Since regular sequences of forms are permutable, we have that $F_{1}, \ldots, F_{h}, x_{1}, \ldots, x_{m}$ is a regular sequence, and since $K$ is infinite we may extend $F_{1}, \ldots, F_{h}, x_{1}, \ldots, x_{m}$ to a homogeneous system of parameters using linear forms, which we may assume are the variables $x_{m+h+1}, \ldots, x_{N}$. That is, $F_{1}, \ldots, F_{h}, x_{1}, \ldots, x_{m}, x_{h+m+1}, \ldots, x_{N}$ form a homogeneous system of parameters for $K\left[x_{1}, \ldots, x_{N}\right]$. The images of $F_{1}, \ldots, F_{h}$ when we kill the variables in this regular sequence are precisely the elements $f_{1}, \ldots, f_{h} \in$ $K\left[u_{1}, \ldots, u_{h}\right]$. Hence, the quadratic forms $f_{1}, \ldots, f_{h}$ are a homogeneous system of parameters for $K\left[u_{1}, \ldots, u_{h}\right]$. In particular, they are linearly independent over $K$. The remaining $F_{i}$ may be permuted so that $f_{1}, \ldots, f_{d}$ is a $K$-vector space basis for the $K$-span of the $f_{i}$. For $i>d$ we may subtract a $K$-linear combination of $F_{1}, \ldots, F_{d}$ from $F_{i}$ to arrange that $f_{i}$ be zero.

When $F_{1}, \ldots, F_{n}$ are written as polynomials in the leading and front variables $x_{1}, \ldots, x_{m}, u_{1}, \ldots, u_{h}$ (with coefficients in the polynomial ring in the remaining variables) there are at most $m+h$ linear terms: the coefficients are linear forms in $x_{m+h+1}, \ldots, x_{N}$. All these coefficients from all of the $F_{i}$ span a $K$-vector space of dimension $r \leq n(m+h)$. We make a linear change of the variables $x_{m+h+1}, \ldots, x_{N}$ so that the variables $x_{m+h+1}, \ldots, x_{m+h+r}$ span this space. These are the primary coefficient variables, which are also denoted $v_{1}, \ldots, v_{r}$.

Next, when $F_{1}, \ldots, F_{n}$ are written as polynomials in the leading, front, and primary coefficient variables $x_{1}, \ldots, x_{m}, u_{1}, \ldots, u_{h}, v_{1}, \ldots, v_{r}$ (with coefficients in the polynomial ring in the remaining variables) there are at most $n(m+h)$ terms that are linear involving one of the $v_{j}$ : the coefficients are linear forms in $x_{m+h+r+1}, \ldots, x_{N}$. All these coefficients from all of the $F_{i}$ span a $K$-vector space of dimension $s \leq$ $n^{2}(m+h)$. We make a linear change of the variables $x_{m+h+1}, \ldots, x_{N}$ so that the variables $x_{m+h+r+1}, \ldots, x_{m+h+r+s}$ span this space. These are the secondary coefficient variables, which we also denote $w_{1}, \ldots, w_{s}$.

Finally, we refer to $x_{m+h+r+1}, \ldots, x_{N}$ as the tail variables. This set is the complement in $\left\{x_{1}, \ldots, x_{N}\right\}$ of the union of leading, front, and primary coefficient variables. We emphasize again that the tail variables include the secondary coefficient variables.

All of the conditions for standard form are now satisfied. We remark that once we have standard form, it is unaffected by permuting $F_{h+1}, \ldots, F_{d}$, or by multiplying by an invertible matrix over $K$, thereby replacing them with linear combinations that have the same $K$-span. 
We show in the next section that if this procedure is then carried through a second time using the forms consisting of all leading, front, primary, and secondary coefficient variables (these are the new leading variables) and the tails, thus producing a second set of tails, then one of two things happens:

(1) The second set of tails generates an ideal of height at most $h-1$ modulo the ideal generated by the new leading variables. (It may also happen that the set of original tails generates an ideal of height at most $h-1$ modulo the ideal generated by the original leading variables, which is an easier case.)

(2) The leading, front, primary, and secondary coefficient variables and the non-zero elements in first set of tails form a regular sequence.

Either condition yields an estimate of what is needed for $B(m, n, h)$. The first condition requires a larger value and leads to the recursive definition given earlier. Details are given in the next section.

\section{Key behavior of standard form and the proof of the main theorems}

Placing $F_{1}, \ldots, F_{m+n}$ and the variables in standard form puts surprising constraints on the forms. In particular, parts (c), and (d) of the Key Lemma below play a central role in the proof of the main results.

Key Lemma. Let $F_{1}, \ldots, F_{m+n}$ consist of quadratic and linear forms in the polynomial ring $R=K\left[x_{1}, \ldots, x_{N}\right]$ which, together with the variables $x_{1}, \ldots, x_{N}$ are assumed to be in standard form. Let all notation and terminology, including $m, n, h, r$, $s, d, f_{i}, g_{i}, u_{i}, v_{i}, w_{i}, P, \pi$, and $\tau$, be as in the preceding section. Let $I$ be the ideal of $R$ generated by $F_{1}, \ldots, F_{m+n}$. Then:

(a) The elements $f_{1}, \ldots, f_{h}$ form a homogenous system of parameters for the ring $K\left[u_{1}, \ldots, u_{h}\right]$, and since

$$
K\left[f_{1}, \ldots, f_{h}\right] \subseteq K\left[f_{1}, \ldots, f_{d}\right]=K\left[f_{1}, \ldots, f_{n}\right] \subseteq K\left[u_{1}, \ldots, u_{h}\right]
$$

the domain $K\left[f_{1}, \ldots, f_{n}\right]$ has Krull dimension $h$, so that $P$ has height $n-h$.

(b) For $1 \leq i \leq n, F_{i}$ uniquely has the form $f_{i}+e_{i}+g_{i}$ where

$$
e_{i} \in K\left[x_{1}, \ldots, x_{m}, \underline{u}, \underline{v}, \underline{w}\right]
$$

and is also in the ideal of this ring generated by $\left(x_{1}, \ldots, x_{m}, \underline{v}\right)$. Hence,

$$
K\left[F_{1}, \ldots, F_{m+n}\right] \subseteq K\left[g_{1}, \ldots, g_{n}, x_{1}, \ldots, x_{m}, \underline{u}, \underline{v}, \underline{w}\right] .
$$

(c) If $H \in P$, then $H\left(g_{1}, \ldots, g_{n}\right)=0$. That is, the tail polynomials satisfy the front relations.

(d) If $i>d$, the $g_{i}=0$, i.e., $F_{i}=e_{i}$ is in the ideal generated by the leading and primary coefficient variables, and in the polynomial ring generated by the leading variables, front variables, and the primary and secondary coefficient variables.

Proof. The statement that $f_{1}, \ldots, f_{h}$ is a homogeneous system of parameters was proved in the fourth paragraph of the subsection on Achieving standard form, and the other assertions in (a) are immediate.

For (b), $e_{i}$ is clearly the sum of all terms in $F_{i}$ not involving only front variables nor only tail variables. From condition (2) of the definition of standard form there are no terms involving only leading variables. The terms in $e_{i}$ that involve some $x_{i}$ 
or $u_{i}$ in degree 1 have coefficients in the span of the $v_{i}$ by the definition of primary coefficient variables. That is,

$$
e_{i}=\sum_{j=1}^{m} L_{j} x_{j}+\sum_{k=1}^{h} L_{k}^{\prime} u_{k}+e_{i}^{\prime},
$$

where the $L_{j}, L_{j}^{\prime}$ are in the $K$-span of $v_{1}, \ldots, v_{r}$ and the terms in $e_{i}^{\prime}$ are quadratic in the $v_{t}$ or linear in the $v_{t}$, and those that are linear in the $v_{t}$ have coefficients in the $K$-span of $w_{1}, \ldots, w_{s}$ by the definition of secondary coefficient variables.

To prove (c), note first that $H$ is a sum of homogeneous elements of $P$ and so may be assumed homogeneous of degree $k$. Since $-f_{i} \equiv e_{i}+g_{i}$ modulo $I$, we have

$$
0=(-1)^{k} H\left(f_{1}, \ldots, f_{n}\right)=H\left(-f_{1}, \ldots,-f_{n}\right) \equiv H\left(e_{1}+g_{1}, \ldots, e_{n}+g_{n}\right) \text { modulo } I \text {, }
$$

so that

$$
H\left(e_{1}+g_{1}, \ldots, e_{n}+g_{n}\right) \in I .
$$

If $H\left(g_{1}, \ldots, g_{n}\right) \neq 0$, we obtain a contradiction by showing that

$$
F_{1}, \ldots, F_{h}, H\left(e_{1}+g_{1}, \ldots, e_{n}+g_{n}\right)
$$

is a regular sequence of length $h+1$ in $R /\left(x_{1}, \ldots, x_{m}\right)$. To this end, it suffices to show that we have a regular sequence modulo $\left(x_{1}, \ldots, x_{m}, v_{1}, \ldots, v_{r}\right)$. Killing the $v_{j}$ kills the $e_{j}$ by (b) above, and so it suffices to show that

$$
f_{1}+g_{1}, \ldots, f_{h}+g_{h}, H\left(g_{1}, \ldots, g_{n}\right)
$$

is a regular sequence in the polynomial ring in the front and tail variables. This is immediate from Lemma 3.2: since regular sequences of forms are permutable, it suffices to show that

$$
H\left(g_{1}, \ldots, g_{n}\right), f_{1}+g_{1}, \ldots, f_{h}+g_{h}
$$

is a regular sequence. Since the first element is non-zero, it is a non-zero divisor involving only the tail variables, and modulo the tail variables the remaining terms become the regular sequence $f_{1}, \ldots, f_{h}$.

Finally, (d) follows from (c) and the fact that $f_{i}=\pi\left(F_{i}\right)=0$ for $i>d$ : we may take $H=T_{i}$, and this gives that $g_{i}=0$. The remaining statements now follow from (b) above.

Proof of Main Theorem 1. If $h=0$, then $F_{1}, \ldots, F_{n}$ are expressible as sums of multiples by linear forms of $x_{1}, \ldots, x_{m}$. All the multipliers together span a vector space of dimension at most $m n$, and so all of $F_{1}, \ldots, F_{n}$ can be expressed in terms of $m+m n$ variables, which include the variables $x_{1}, \ldots, x_{m}$.

We now assume $h \geq 1$ and use induction on $h$. We may assume standard form. We consider two cases, (1) and (2). Case (2) has subcases (2a) and (2b). Note that Case (1) is, in a sense, subsumed in Case (2a). Let $h^{\prime}$ be the height of the ideal generated by the tail polynomials modulo the secondary coefficient variables. Of course, $h^{\prime} \leq h$.

Case 1: $h^{\prime} \leq h-1$. By the induction hypothesis, we may work in the polynomial ring in the tail variables $\underline{z}$ with the sequence of tail polynomials and secondary coefficient variables $\underline{w}$ to select at most $k \leq B\left(n^{2}(m+h), n, h-1\right)$ tail variables $z_{j_{1}}, \ldots, z_{j_{k}}$, such that $K\left[g_{1}, \ldots, g_{n}, w_{1}, \ldots, w_{s}\right]$ is contained in the $K$-algebra generated by these $z_{j_{\nu}}$ and a sequence of at most $h-1$ distinct quadratic forms that, together with the 
$z_{j_{\nu}}$ form a regular sequence. When we expand this sequence to include the leading, front, and primary coefficient variables (all these variables are disjoint from the tail variables), we still have a regular sequence, and by part (b) of the Key Lemma, the algebra these generate contains $K\left[F_{1}, \ldots, F_{m+n}\right]$. In this case the constraint placed on $B(m, n, h)$ is that it be at least

$$
m+h+n(m+h)+B\left(n^{2}(m+h), n, h-1\right) .
$$

Case 2: $h^{\prime}=h$. Let $S$ be the polynomial ring over $K$ generated by the tail variables. We work with sequence $g_{1}, \ldots, g_{n}, w_{1}, \ldots, w_{s}$ in $S$. We may now put these in standard form. We can do so without changing $g_{1}, \ldots, g_{h}$ : we claim these already form a regular sequence of length $h$ modulo the secondary coefficient variables. (To meet the condition that an initial segment of the new front polynomials be independent over $K$ while the rest are zero, we may adjust $F_{h+1}, \ldots, F_{d}$ by multiplying by an invertible matrix over $K$ : note the comment on p. 7.) The reason is that there is a homomorphism of $K$-algebras $K\left[f_{1}, \ldots, f_{n}\right] \rightarrow K\left[g_{1}, \ldots, g_{n}\right]$ sending $f_{i} \mapsto g_{i}$ for all $i$. This is well-defined precisely because of part (d) of the Key Lemma: the $g_{i}$ satisfy all the relations in $P$ on the $f_{i}$. It is clearly surjective. it follows that $K\left[g_{1}, \ldots, g_{n}\right]$ is module-finite over $K\left[g_{1}, \ldots, g_{h}\right]$. But $K\left[g_{1}, \ldots, g_{n}\right]$ maps onto the new ring of front polynomials, which will have Krull dimension $h$. Thus, $K\left[g_{1}, \ldots, g_{h}\right]$ must have dimension $h$, and no less. This implies that $g_{1}, \ldots, g_{h}$ is a regular sequence, even modulo the secondary coefficient variables. Thus, the prime ideal of relations on $g_{1}, \ldots, g_{n}$ must be exactly $P$. The same holds for the new ideal of front relations on the front polynomials of the $g_{i}$. We let $\alpha_{1}, \ldots, \alpha_{n}$ denote the new front polynomials and let $\beta_{1}, \ldots, \beta_{n}$ denote the new tail polynomials. Let $h^{\prime \prime}$ denote the height of the ideal generated by the new tail polynomials in the ring generated over $K$ by the new tail variables modulo the ideal generated by the new secondary coefficient variables.

Subcase (2a): $h^{\prime \prime} \leq h-1$. This is very much like Case 1. We have that $K\left[F_{1}, \ldots, F_{m+n}\right]$ is contained in the polynomial ring generated over $K$ by the original leading, front, primary coefficient variables, secondary coefficient variables, and the original tail polynomials. But $K\left[w_{1}, \ldots, w_{s}, g_{1}, \ldots, g_{n}\right]$ is contained in turn in the $K$ algebra generated by the secondary coefficient variables (which are also the new leading variables), the new front, new primary coefficient, and new secondary coefficient variables, and the new tails. We count variables as follows:

\begin{tabular}{ll} 
Type of variable or term & Cardinality \\
\cline { 1 - 2 } leading & $m$ \\
front & $h$ \\
primary coefficient & $(m+h) n$ \\
secondary coefficient = new leading & $(m+h) n^{2}$ \\
new front & $h$ \\
new primary coefficient & $\left((m+h) n^{2}+h\right) n$ \\
new secondary coefficient and new tails & covered by $B\left((m+h) n^{2}, n, h-1\right)$
\end{tabular}

In this case the constraint placed on $B(m, n, h)$ is that it will be at least

$$
m+h+(m+h) n+(m+h) n^{2}+h+\left((m+h) n^{2}+h\right) n+B\left((m+h) n^{2}, n, h-1\right)
$$


which may be rewritten as

$$
(m+h)\left(n^{3}+n^{2}+n+1\right)+h(n+1)+B\left((m+h) n^{2}, n, h-1\right) .
$$

Since this is larger than what was needed in Case 1 , we no longer need to consider Case 1. This is the formula used in the recursive definition of $B(m, n, h)$.

Subcase (2b): $h^{\prime \prime}=h$. In this case we show that $d=h$. To see this, note that the the relations on $g_{1}, \ldots, g_{n}$ are exactly given by $P$, and because $h^{\prime}=h$ we know that the relations on $\alpha_{1}, \ldots, \alpha_{n}$ are exactly given by $P$ again. But the elements $\beta_{1}, \ldots, \beta_{n}$ are homomorphic images of the elements $g_{1}, \ldots, g_{n}$, so that these satisfy $P$, and so are the elements $\alpha_{1}+\beta_{1}, \ldots, \alpha_{n}+\beta_{n}$, which must also satisfy $P$. Moreover, since $h^{\prime \prime}=h$, the algebra generated by the elements $\beta_{1}, \ldots, \beta_{n}$ has dimension $h$, and $P$ gives the ideal of all relations on the elements $\beta_{1}, \ldots, \beta_{n}$. We may apply Lemma 3.4 to conclude that the $P$ is generated by linear forms. Since standard form was set up so that $f_{1}, \ldots, f_{d}$ are linearly independent but the $f_{i}$ for $i>h$ are integral over $K\left[f_{1}, \ldots, f_{h}\right]$, we must have that $f_{i}=0$ for $i>h$, i.e., $d=h$ as claimed. Hence, the only non-zero tail polynomials are $g_{1}, \ldots, g_{h}$, which form a regular sequence modulo $x_{1}, \ldots, x_{m}, \underline{u}, \underline{v}, \underline{w}$. Then $K\left[F_{1}, \ldots, F_{m+n}\right] \subseteq K\left[g_{1}, \ldots, g_{h}, x_{1}, \ldots, x_{m}, \underline{u}, \underline{v}, \underline{w}\right]$, and the only constraint on $B(m, n, h)$ is that it will be at least $(m+h)\left(1+n+n^{2}\right)$. Therefore, using the formula obtained in Subcase (2a) will cover all cases.

\section{Size estimates}

We study briefly the size of the functions $B(m, n, h), C_{0}(s)$, and $C(s)$. We shall use the alternative notation $B(m, n, h)=B_{h}(m, n)=B_{h}$. Let

$$
g=g(n)=n^{3}+n^{2}+n+1
$$

and let

$$
g_{1}=g_{1}(n)=g(n)+n+1
$$

Thus, $g$ and $g_{1}$ are both monic in $n$ of degree 3 . Let $\theta_{h}(t)=\sum_{i=0}^{h-1} t^{i}$, so that $\theta_{0}=0$ and $\theta_{h}=t \theta_{h-1}+1$ for $h \geq 1$. We claim that for all $h$,

$$
(m+h)\left((n+1)\left(n^{2}\right)^{h}+g \theta_{h}\left(n^{2}\right)\right)
$$

is a lower bound for $B_{h}$, while

$$
(m+h)\left((n+1)\left(n^{2}+1\right)^{h}+g_{1} \theta_{h}\left(n^{2}+1\right)\right)
$$

is an upper bound. Note that the two agree with $B_{0}$ when $h=0$. The proof that the given expressions are bounds is by induction on $h$. If we assume the estimates for a given $h-1 \geq 0$, we have

$$
\begin{aligned}
B_{h}= & (m+h) g+h(n+1)+B_{h-1}\left((m+h) n^{2}, n\right) \\
\leq & (m+h) g+(m+h)(n+1)+\left((m+h) n^{2}+h-1\right)\left((n+1)\left(n^{2}+1\right)^{h-1}\right. \\
& \left.+g_{1} \theta_{h-1}\left(n^{2}+1\right)\right) .
\end{aligned}
$$


We may replace the summand $h-1$ in the first factor of the third term by $m+h$. The expression then becomes

$$
(m+h)\left(g_{1}+\left(n^{2}+1\right)\left((n+1)\left(n^{2}+1\right)^{h-1}+g_{1} \theta_{h-1}\left(n^{2}+1\right)\right),\right.
$$

which simplifies to the required form. We also have

$$
B_{h} \geq(m+h) g+h(n+1)+\left((m+h) n^{2}+(h-1)\right)\left((n+1)\left(n^{2}\right)^{h-1}+g \theta_{h-1}\left(n^{2}\right)\right) .
$$

We may simply drop the terms $h(n+1)$ and $h-1$ to get the required estimate.

A lower bound for both $C_{0}(s)$ and for $C(s)$ may be obtained from the case $m=0$, $s=n, h=s-1$, which yields

$$
C(s) \geq(s-1)\left((s+1)\left(s^{2}\right)^{s-1}+g(s) \theta_{s-1}\left(s^{2}\right)\right)+(s-1) .
$$

Since whenever $h=s$ we must have $m=0$ and that the given forms are already a regular sequence, we may assume $h \leq s-1$. This yields the upper bound

$$
C(s) \leq(s-1)\left((s+1)\left(s^{2}+1\right)^{s-1}+g_{1}(s) \theta_{s-1}\left(s^{2}+1\right)\right)+(s-1) .
$$

Both bounds are asymptotic to $2 s^{2 s}$, since $\theta_{s-1}(t)=\left(t^{s-1}-1\right) /(t-1)$, and $\left(s^{2}+1\right)^{s-1}$ is asymptotic to $\left(s^{2}\right)^{s-1}$. Hence, both $C_{0}(s)$ and $C(s)$ are asymptotic to $2 s^{2 s}$.

\section{The question in higher degree}

We raise here the following question: is there an integer $C(n, d)$ such that given $n$ forms $F_{1}, \ldots, F_{n}$ of degree at most $d$ in a polynomial ring $R$ over an infinite field $K$, there exists a regular sequence of forms $G_{1}, \ldots, G_{k} \in R$ of degree at most $d$ with $k \leq C(n, d)$ such that $F_{1}, \ldots, F_{n} \in K\left[G_{1}, \ldots, G_{k}\right]$ ? Our main result here is, of course, the case $d=2$. An affirmative answer would yield results analogous to our main theorems for arbitrary $d: C(n, d)$ would bound the projective dimension of $R /\left(F_{1}, \ldots, F_{n}\right)$ over any field, and $C(d n, d)$ would give corresponding results when the $F_{i}$ are polynomials of degree at most $d$, not required to be homogeneous.

\section{Acknowledgments}

The second author was partially supported by a grant from the National Science Foundation (DMS-0901145).

\section{References}

[1] J. Beder, J. McCullough, L. Nunez-Betancourt, A. Seceleanu, B. Snapp and B. Stone, Ideals with larger projective dimension and regularity, preprint, 10pp., arXiv:1101.3368v1 [math.AC].

[2] L. Burch, A note on the homology of ideals generated by three elements in local rings, Proc. Cambridge Philos. Soc. 64 (1968), 949-952.

[3] G. Caviglia and M. Kummini, Some ideals with large projective dimension, Proc. Amer. Math. Soc. 136(2) (2008), 505-509.

[4] B. Engheta, Bounds on projective dimension, Ph.D. thesis, University of Kansas, 2005.

[5] B. Engheta, A bound on the projective dimension of three cubics, J. Symbolic Comput. 45(1) (2010), 60-73.

[6] B. Engheta, On the projective dimension and the unmixed part of three cubics, J. Algebra 316(2) (2007), 715-734.

[7] P. Kohn, Ideals generated by three elements, Proc. Amer. Math. Soc. 35 (1972), 55-58.

[8] H. Matsumura, Commutative algebra, W.A. Benjamin, Inc., New York, 1970. 
[9] J. McCullough, A family of ideals with few generators in low degree and large projective dimension, Proc. Amer. Math. Soc. 139 (2011), 2017-2023.

[10] I. Peeva and M. Stillman, Open problems on syzygies and Hilbert functions, J. Commut. Algebra 1(1), (2009), 159-195.

Department of Mathematics, University of Michigan, 530 Church Street, Ann Arbor, MI 48109-1043, USA

E-mail address: hochster@umich.edu

Altair Engineering, 1820 Big Beaver Rd, Troy, MI 48083, USA

E-mail address: antigran@umich.edu 